\title{
Aflatoxin B1 degradation during co-cultivation of Aspergillus flavus and Pleurotus ostreatus strains on rice straw
}

\author{
Arijit Das - Sourav Bhattacharya - Muthusamy Palaniswamy • \\ Jayaraman Angayarkanni
}

Received: 28 March 2014/Accepted: 27 May 2014/Published online: 11 June 2014

(c) The Author(s) 2014. This article is published with open access at Springerlink.com

\begin{abstract}
Aflatoxin B1 (AFB1) produced by Aspergillus flavus is known to have carcinogenic and teratogenic effects on animal health. Accidental feeding of AFB1contaminated rice straw may be detrimental to dairy cattle. White-rot basidiomycetous fungus Pleurotus ostreatus can grow on different agronomic wastes by synthesizing different ligninolytic enzymes. These extracellular enzymes are capable of degrading many environmentally hazardous compounds including AFB1. The present study examines the ability of different strains of $P$. ostreatus to degrade AFB1 in contaminated rice straw. Different strains of $A$. flavus were inoculated on rice straw for AFB1 production. The moldy straw was then subjected to co-cultivation by different strains of $P$. ostreatus. The extent of AFB1 degradation was determined by high performance liquid chromatography. Results indicated the presence of AFB1 in the moldy straw samples at levels of $27.95 \pm 0.23$ and $21.26 \pm 0.55 \mu \mathrm{g} / \mathrm{g}$ of dry substrate for A. flavus MTCC 2798 and A. flavus GHBF09, respectively. Co-cultivation of $P$. ostreatus strains on AFB1-contaminated rice straw revealed their ability to rapidly colonize the substrate by profuse hyphal ramification. Highest degradation of AFB1 $(89.41 \%)$ was recorded in the straw containing co-cultures
\end{abstract}

\footnotetext{
A. Das $(\bowtie) \cdot$ S. Bhattacharya $\cdot$ M. Palaniswamy Department of Microbiology, Karpagam University, Coimbatore 641021, Tamil Nadu, India e-mail: jit2007das@gmail.com
}

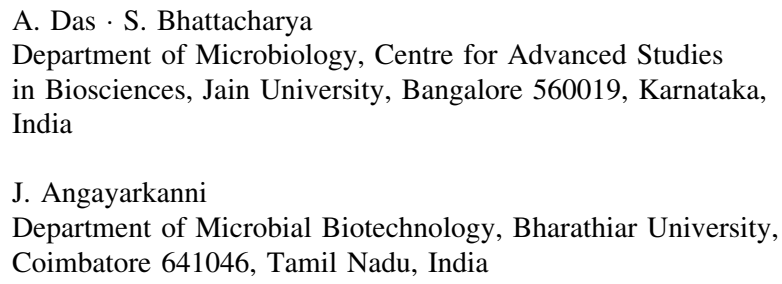

of A. flavus MTCC 2798 and P. ostreatus GHBBF10. Natural isolate $P$. ostreatus GHBBF10 demonstrated higher AFB1-degradation potential than $P$. ostreatus MTCC 142. This basidiomycete strain can be further exploited to effectively degrade moderate concentrations of AFB1 in contaminated moldy rice straw.

Keywords Aflatoxin B1 Pleurotus ostreatus . Co-cultivation $\cdot$ Rice straw

\section{Introduction}

Mycotoxins are highly toxic fungal metabolites which have adverse effects on human and animal health. Among the mycotoxins, aflatoxin B1 (AFB1) is extremely hazardous for being mutagenic, carcinogenic and teratogenic (Khoshpey et al. 2011). AFB1 is produced by strains of Aspergillus flavus and A. parasiticus on a variety of agricultural commodities. Animal feeds and forages may become contaminated with AFB1 in the fields and/or during storage under conditions conducive for fungal growth (Streit et al. 2012). In the tropical countries like India, rice (paddy) straw is often used as cattle feed. However, consumption of AFB1-contaminated straw can lead to aflatoxicosis in cattle and eventually lead to transmission of these toxins or their metabolites into the milk of dairy animals (Bennett and Klich 2003).

White-rot basidiomycetous fungus such as Pleurotus ostreatus is able to transform AFB1 into less toxic metabolites (Guan et al. 2008). Under natural condition, $P$. ostreatus grows on dead wood but can be easily cultivated on different agro-industrial wastes (Sánchez 2010). It produces different oxidative [laccase and manganese peroxidase (MnP)] and hydrolytic (cellulase, xylanase and 
tannase) enzymes which are involved in utilization of lignocellulosic substrates (da Luz et al. 2012).

Degradation of AFB1 by white-rot fungi through the production of extracellular lignin modifying enzymes was previously investigated (Cohen et al. 2002). Due to lack of substrate specificity, laccase can degrade many xenobiotic compounds. A correlation between laccase activity and AFB1 degradation was previously reported by Alberts et al. (2009). Though some studies on AFB1 degradation have been conducted, very few scientific literatures are available on AFB1 degradation by $P$. ostreatus in A. flavus-contaminated moldy rice straw. Among $P$. ostreatus, different strains may vary in their ability to degrade AFB1. Selection of a potent basidiomycete strain may facilitate effective degradation of this hazardous toxin. Therefore, the present study was conducted to assess the ability of different strains of $P$. ostreatus to degrade AFB1 in contaminated rice straw.

\section{Materials and methods}

Chemicals and reagents

AFB1 standard was procured from Sigma (USA). All other analytical grade laboratory chemicals were purchased from S D Fine-Chem Ltd. (Mumbai, India) and Nice Chemicals (Kochi, India).

Source of fungal strains

A. flavus MTCC 2798 and P. ostreatus MTCC 142 were obtained from Microbial Type Culture Collection and Gene Bank, Chandigarh, India. An aflatoxigenic fungal strain identified as A. flavus GHBF09 (GenBank accession number KC987360) was isolated from rhizosphere soil (Das et al. 2013a). A basidiomycetous fungal strain identified as $P$. ostreatus GHBBF10 (GenBank accession number KC987361) was isolated from a decomposing tree trunk (Das et al. 2013b). Cultures of A. flavus and P. ostreatus were maintained on potato dextrose agar and glucose yeast extract agar plates, respectively.

\section{Substrate preparation}

Rice (paddy) straw was obtained from the local market in Bangalore. The straw was washed several times under running tap water, dried in hot air oven at $80{ }^{\circ} \mathrm{C}$ and cut into approximately $1 \mathrm{~cm}$ pieces. This was used as the substrate for co-cultivation. Dry straw (1 g) was filled in a glass culture tube measuring $2.5 \mathrm{~cm}$ in diameter and $15 \mathrm{~cm}$ long, moistened with $5 \mathrm{ml}$ supplemented mineral salt solution containing (g/l) lactose, 10; yeast extract, 5;
$\mathrm{KH}_{2} \mathrm{PO}_{4}, 0.2 ; \mathrm{MgSO}_{4} \cdot 7 \mathrm{H}_{2} \mathrm{O}, 0.1 ; \mathrm{NH}_{4} \mathrm{Cl}, 0.3 ; \mathrm{CaCO}_{3}, 1.0$ and distilled water, at initial $\mathrm{pH} 5.8$ and autoclaved (Das et al. 2013b).

\section{Co-cultivation of A. flavus and P. ostreatus strains}

Degradation of AFB1 during co-cultivation of $A$. flavus and $P$. ostreatus strains on rice straw was investigated. Duplicate sets of four culture tubes containing the substrate were labeled. Two tubes were inoculated with spore suspension of A. flavus MTCC 2798 and two other tubes with A. flavus GHBF09. The inoculated sets of tubes were incubated at $28 \pm 2{ }^{\circ} \mathrm{C}$ for 15 days for AFB1 production. Post incubation, one set of tubes was subjected to AFB1 analysis using high performance liquid chromatography (HPLC). In the second set, two alternate tubes containing moldy straw with live A. flavus cultures were aseptically inoculated with two mycelial plugs of $P$. ostreatus MTCC 142, while the other two tubes were inoculated with $P$. ostreatus GHBBF10. This set of inoculated tubes was incubated at $30 \pm 2{ }^{\circ} \mathrm{C}$ for 15 days in dark. Mycelial growth in these tubes was carefully monitored daily. After 15 days of incubation, the level of residual AFB1 present in the tubes was determined by HPLC. A sterile uninoculated tube containing the substrate was maintained separately throughout the study for sterility check.

\section{Extraction of AFB1}

The extraction of AFB1 was performed using AOAC protocols (AOAC International 2000) with few modifications. Following incubation, the substrate colonized with fungal mycelia was homogenized in a blender with $20 \mathrm{ml}$ of $90 \%(\mathrm{v} / \mathrm{v})$ methanol and filtered through coarse filter paper. To the filtrate equal volume of methylene chloride was added and AFB1 was partitioned by shaking vigorously for $15 \mathrm{~min}$. The extraction procedure was repeated with the aqueous fraction. The methylene chloride fractions were pooled and concentrated in hot air oven at $40{ }^{\circ} \mathrm{C}$ overnight.

\section{Analysis of AFB1 using HPLC}

The concentrated residue was reconstituted in $1 \mathrm{ml}$ of acetonitrile and filtered through $0.25 \mu$ nylon membrane filter (Axiva Sichem Biotech, Delhi, India). The working standard solution of AFB1 (concentration of $10 \mu \mathrm{g} / \mathrm{ml}$ ) was prepared using acetonitrile. Working standard $(20 \mu \mathrm{l})$ containing $0.2 \mu \mathrm{g}$ of standard AFB1 was injected into the HPLC system (Waters, USA, model number-2487, with Dual $\lambda$ absorbance UV detector and binary pump system, model number-1525). A reverse phase, YMCA Triart C18 column $(150 \times 4.6 \mathrm{~mm}, 3 \mu \mathrm{m})$ was used. The mobile phase 
consisted of acetonitrile-methanol-water (1:1:2, v/v/v). A total run time of 14 min was maintained at a flow rate of $0.8 \mathrm{ml} / \mathrm{min}$ under ambient temperature. The absorbance of AFB1 was determined at $360 \mathrm{~nm}$. Area under AFB1 absorbance peak was used to estimate its concentration and also the percentage of degradation using the following formula (Guan et al. 2010):

Percentage of AFB1 degradation $=\left[\left(C_{\mathrm{i}}-C_{\mathrm{f}}\right) / C_{\mathrm{i}}\right] \times 100$

where, $C_{\mathrm{i}}$ is the initial concentration of AFB1 and $C_{\mathrm{f}}$ is the final concentration of AFB1.

\section{Statistical analysis}

The experiment was conducted in triplicate. The data were analyzed using single factor analysis of variance (ANOVA) and graphically presented as mean $\pm \mathrm{SD} \quad(n=3)$. ANOVA was performed using Microsoft Excel 2007. $P$ values $<0.05$ were considered significant with a confidence limit of $95 \%$.

\section{Results and discussion}

In the present study, co-cultivation of A. flavus and P. ostreatus strains on rice straw was carried out to understand the practical applicability of the basidiomycetous fungus in AFB1 degradation in rice straw which may be contaminated with AFB1 in agricultural fields.

Growth of A. flavus strains on rice straw and AFB1 production

After 15 days of incubation, rice straw inoculated with the tested strains of A. flavus turned completely moldy due to profuse mycelial growth and sporulation of the fungi (Fig. 1). HPLC analysis of these moldy straw samples revealed the presence of $\mathrm{AFB} 1$ at levels of $27.95 \pm 0.23$

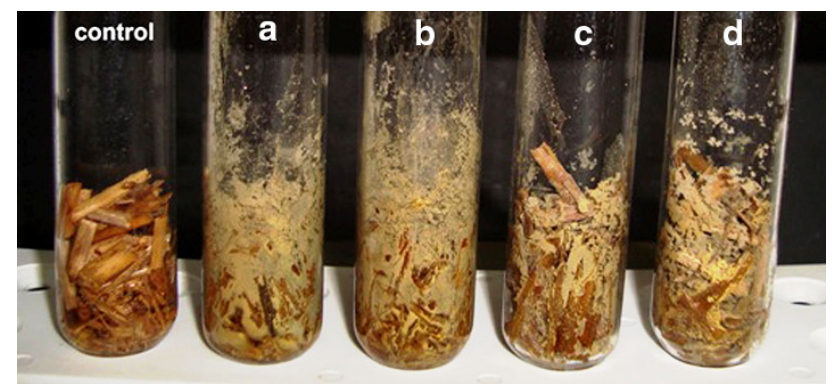

Fig. 1 Mycelial growth of A. flavus in tubes containing moist rice straw as substrate. $a, b$ Tubes inoculated with A. flavus MTCC 2798. $c, d$ Tubes inoculated with A. flavus GHBF09. A sterile uninoculated tube (control) is shown on the left and $21.26 \pm 0.55 \mu \mathrm{g} / \mathrm{g}$ of dry substrate for A. flavus MTCC 2798 and A. flavus GHBF09, respectively. Comparatively, A. flavus MTCC 2798 demonstrated higher production of AFB1. Zain (2011) suggested that among various factors, the ability to produce AFB1 in foods and feeds is also dependent on fungal strain specificity and strain variation. Moreover, the presence of elevated moisture content and high $\mathrm{C} / \mathrm{N}$ ratio in rice straw would have facilitated increased AFB1 production from the tested strains of $A$. flavus (Abdulla 2007).

Growth of $P$. ostreatus strains on moldy rice straw

The growth of oyster mushroom generally depends on the type of substrate used for its cultivation. Generally, rice straw supports higher yields of oyster mushrooms compared to wheat straw under identical cultivation conditions (Zhang et al. 2002). The presence of high initial moisture content $(83 \%)$ within the straw fibers together with utilizable lignin and cellulose would have stimulated abundant mycelial growth of $P$. ostreatus through the synthesis of lignocellulolytic enzymes like laccase and cellulase. An earlier study reported larger fruiting bodies in P. eryngii when cultivated on rice straw (Moonmoon et al. 2010).

Co-cultivation of $P$. ostreatus strains on rice straw already containing $A$. flavus culture revealed the ability of $P$. ostreatus strains to rapidly colonize the substrate. Following incubation, the tubes inoculated with $P$. ostreatus MTCC 142 showed moderate hyphal proliferation, whereas, the tubes inoculated with $P$. ostreatus GHBBF10 demonstrated profuse mycelial growth (Fig. 2). This basidiomycete strain might have synthesized higher titres of extracellular enzymes, which would have resulted in better utilization of the lignocellulosic substrate.

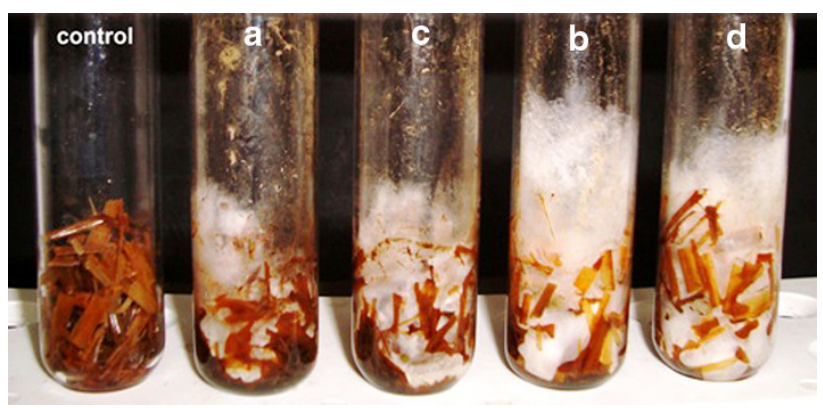

Fig. 2 Tubes containing moist rice straw with co-cultures of A. flavus and $P$. ostreatus strains. $a$ Tube inoculated with A. flavus MTCC $2798+P$. ostreatus MTCC 142. $b$ Tube inoculated with A. flavus MTCC $2798+P$. ostreatus GHBBF10. $c$ Tube inoculated with $A$. flavus $\mathrm{GHBF} 09+P$. ostreatus MTCC 142. $d$ Tube inoculated with $A$. flavus $\mathrm{GHBF} 09+P$. ostreatus GHBBF10. A sterile uninoculated tube (control) is shown on the left 
Fig. 3 HPLC chromatograms showing AFB1 peaks a peak for AFB1 standard, b AFB1 peak after degradation by $P$. ostreatus MTCC 142, c AFB1 peak after degradation by $P$. ostreatus GHBBF10. AFB1 peaks have been indicated by arrows
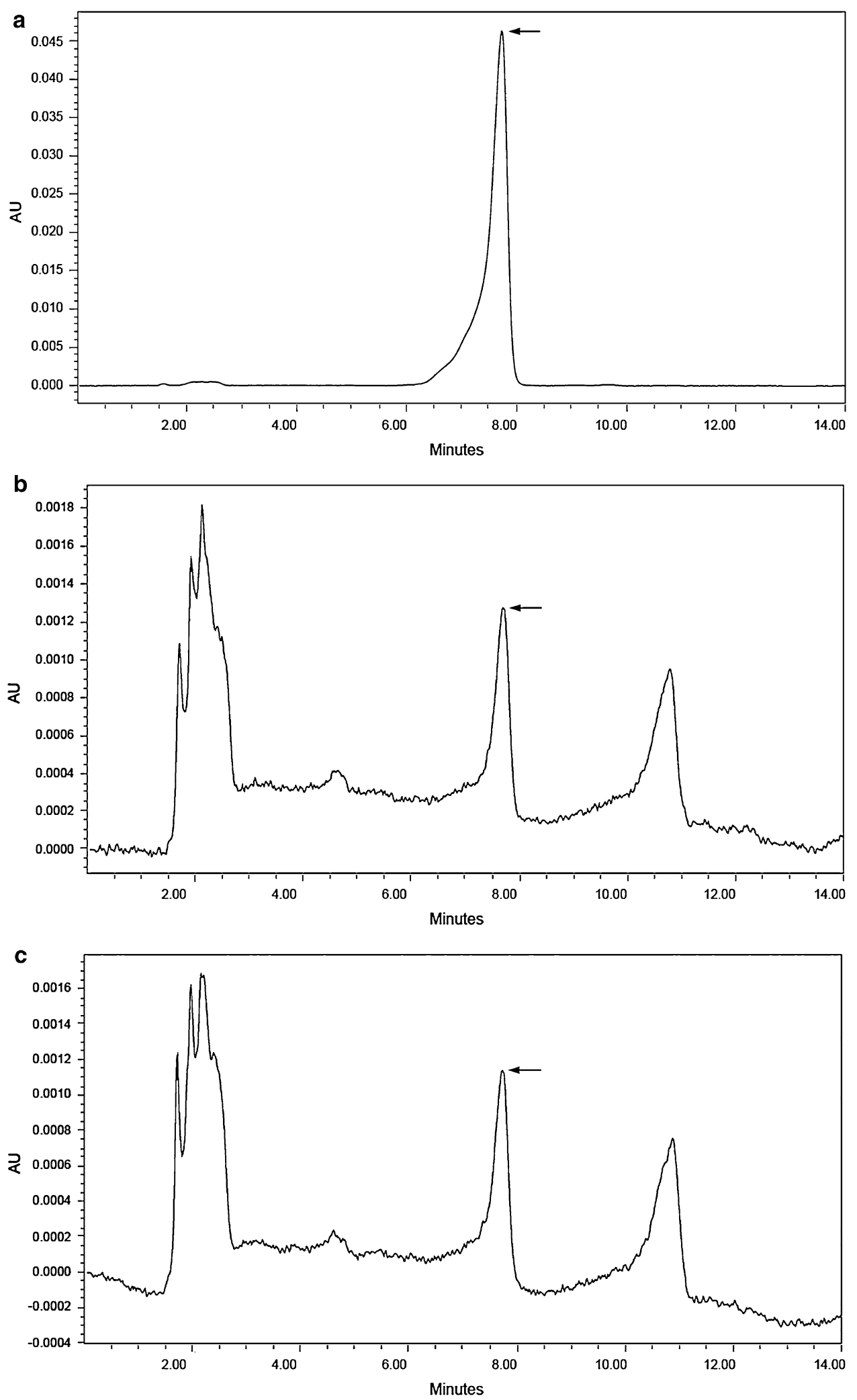
AFB1 degradation during co-cultivation of fungal strains

HPLC chromatogram revealed the peak for AFB1 standard at retention time of $7.722 \mathrm{~min}$ (Fig. 3a). The chromatograms for residual AFB1 after degradation by $P$. ostreatus MTCC 142 and P. ostreatus GHBBF10 have been illustrated in Fig. $3 \mathrm{~b}$ and c, respectively. From the chromatograms it became evident that AFB1 was still present in the samples after co-cultivation, but at reduced concentrations. The presence of several peaks other than that for AFB1 may explain the possible formation of AFB1 adducts and various intermediate compounds during AFB1 degradation (Das et al. 2014). The highest degradation of AFB1 (89.41\%) was recorded in the tube containing co-cultures of A. flavus MTCC 2798 and $P$. ostreatus GHBBF10, with residual AFB1 content of $2.95 \pm 0.03 \mu \mathrm{g} / \mathrm{g} \quad$ (Fig. 4). The lowest degradation $(81.73 \%)$ was noted in the tube containing co-cultures of A. flavus GHBF09 and P. ostreatus MTCC 142, with residual AFB1 content of $3.88 \pm 0.01 \mu \mathrm{g} / \mathrm{g}$. Comparatively, higher percentages of AFB1 degradation were recorded in substrate inoculated with the natural isolate $P$. ostreatus GHBBF10. This could be attributed to its actively functional degradative enzymes' synthetic machinery owing to its natural isolation from decomposing tree trunk containing lignin. In a previous study on AFB1 degradation in contaminated rice straw by $P$. ostreatus strains, we reported that the natural isolate $P$. ostreatus GHBBF10 secreted higher titres of laccase (1.95 U/gds) and MnP (2.84 U/gds), compared to $P$. ostreatus MTCC 142 (Das et al. 2014). Degradation of recalcitrant compounds by $P$. ostreatus is mediated by secretion of degradative enzymes like laccase and $\mathrm{MnP}$

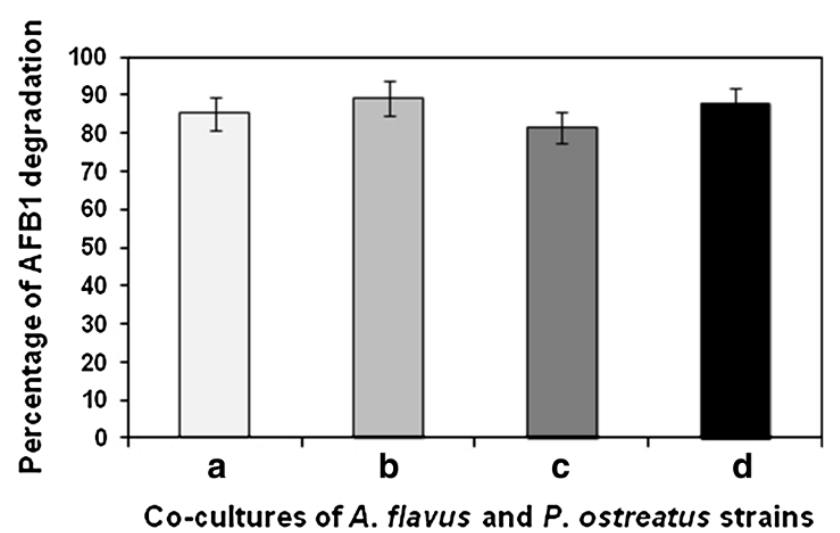

Fig. 4 Extent of AFB1 degradation during co-cultivation of fungal strains on rice straw. a Straw containing A. flavus MTCC $2798+$ $P$. ostreatus MTCC 142, $b$ straw containing A. flavus MTCC $2798+$ $P$. ostreatus $\mathrm{GHBBF} 10, c$ straw containing A. flavus $\mathrm{GHBF} 09+P$. ostreatus MTCC 142, $d$ straw containing A. flavus $\mathrm{GHBF} 09+P$. ostreatus GHBBF10. Data represent mean $\pm \mathrm{SD}(n=3) ; P<0.05$
(Bhattacharya et al. 2014). Furthermore, analysis of AFB1 degradation by HPLC and liquid chromatography mass spectrometry revealed reduction in initial concentration of AFB1 and formation of decarbonylated and $O$-dealkylated degradation compounds, respectively (Das et al. 2014).

Due to profuse ramification of the basidiomycete mycelia, the straw containing A. flavus MTCC 2798 was effectively colonized and the synthesized AFB1, though present at highest concentration $(27.95 \pm 0.23 \mu \mathrm{g} / \mathrm{g})$, was degraded to a greater extent. This observation may be further explained by the presence of yeast extract as nitrogen source in the supplemented mineral salt solution which probably enhanced the laccase production. Gomes et al. (2009) had reported that supplementation of nitrogen in rice straw resulted in 3-4 times increased production of laccase in lignocellulolytic fungi.

In a previous study, co-cultivation of A. flavus and $P$. ostreatus was carried out on various substrates. On wheat straw, corn cobs and millet, A. flavus produced aflatoxin after 3 weeks of cultivation. A subsequent cultivation of $P$. ostreatus on A. flavus-contaminated straw led to detoxification of the straw and corn cobs. It was found that $P$. ostreatus could liquidate colonies of A. flavus. However, cultivation of $P$. ostreatus in the presence of $40-100 \mu \mathrm{g}$ of AFB1/g of substrate did not result in complete detoxification of the material even after 34 days of co-cultivation, but AFB1 concentration decreased to about one-fourth of the added amount (Ginterová et al. 1980).

The present findings are significant as biodegradation of AFB1 with microorganisms or their enzymes may be considered as the best strategy for detoxification of contaminated feedstuffs. This approach is considered as environment friendly in contrast to physico-chemical techniques of detoxification (Upadhaya et al. 2010).

\section{Conclusions}

From this co-cultivation study, it might be deduced that both standard and naturally isolated strains of $P$. ostreatus could effectively degrade moderate concentrations of AFB1 in A. flavus-contaminated moldy rice straw. Further studies would be required to enhance the AFB1-degradation potential of the basidiomycete strains through process optimization.

Acknowledgments We wish to extend our sincere gratitude to the managements of Karpagam University, Jain University and Bharathiar University for their encouraging support. We express our heartfelt thanks to Mrs. K. Prashanthi, Jain University, Bangalore, for her assistance in HPLC analysis.

Conflict of interest The authors declare that they have no conflict of interest. 
Open Access This article is distributed under the terms of the Creative Commons Attribution License which permits any use, distribution, and reproduction in any medium, provided the original author(s) and the source are credited.

\section{References}

Abdulla HM (2007) Enhancement of rice straw composting by lignocellulolytic actinomycete strains. Int $\mathrm{J}$ Agric Biol 9:106-109

Alberts JF, Gelderblom WC, Botha A, van Zyl WH (2009) Degradation of aflatoxin $\mathrm{B}(1)$ by fungal laccase enzymes. Int $\mathbf{J}$ Food Microbiol 135:47-52

AOAC International (2000) Official methods of analysis of AOAC International, 17th edn. Association of Analytical Communities, Gaithersburg

Bennett JW, Klich M (2003) Mycotoxins. Clin Microbiol Rev $16: 497-516$

Bhattacharya S, Das A, Prashanthi K, Palaniswamy M, Angayarkanni J (2014) Mycoremediation of benzo[a]pyrene by Pleurotus ostreatus in the presence of heavy metals and mediators. 3 Biotech 4:205-211

Cohen R, Persky L, Hadar Y (2002) Biotechnological applications and potential of wood-degrading mushrooms of the genus Pleurotus. Appl Microbiol Biotechnol 58:582-594

da Luz JM, Nunes MD, Paes SA, Torres DP, de Cássia Soares da Silva M, Kasuya MC (2012) Lignocellulolytic enzyme production of Pleurotus ostreatus growth in agroindustrial wastes. Braz J Microbiol 43:1508-1515

Das A, Bhattacharya S, Palaniswamy M, Angayarkanni J (2013a) Molecular identification of Aspergillus flavus GHBF09 involved in aflatoxin B1 production in rice straw. Ann Biol Res 4:102-110

Das A, Bhattacharya S, Palaniswamy M, Angayarkanni J (2013b) Assessment of parameters influencing rice straw associated mycelia growth of Pleurotus ostreatus MTCC 142 and a wild isolate of Pleurotus ostreatus. Int Res J Biological Sci 2:15-21
Das A, Bhattacharya S, Palaniswamy M, Angayarkanni J (2014) Biodegradation of aflatoxin B1 in contaminated rice straw by Pleurotus ostreatus MTCC 142 and Pleurotus ostreatus GHBBF10 in the presence of metal salts and surfactants. World J Microbiol Biotechnol. doi:10.1007/s11274-014-1657-5

Ginterová A, Polster M, Janotková O (1980) The relationship between Pleurotus ostreatus and Aspergillus flavus and the production of aflatoxin. Folia Microbiol (Praha) 25:332-336

Gomes E, Aguiar AP, Carvalho CC, Bonfá MRB, da Silva R, Boscolo M (2009) Ligninases production by basidiomycetes strains on lignocellulosic agricultural residues and their application in the decolorization of synthetic dyes. Braz J Microbiol 40:31-39

Guan S, Ji C, Zhou T, Li J, Ma Q, Niu T (2008) Aflatoxin B 1 degradation by Stenotrophomonas maltophilia and other microbes selected using coumarin medium. Int $\mathrm{J}$ Mol Sci 9:1489-1503

Guan S, Zhao L, Ma Q, Zhou T, Wang N, Hu X, Ji C (2010) In vitro efficacy of Myxococcus fulvus ANSM068 to biotransform aflatoxin $\mathrm{B}_{1}$. Int J Mol Sci 11:4063-4079

Khoshpey B, Farhud DD, Zaini F (2011) Aflatoxins in Iran: nature, hazards and carcinogenicity. Iran J Public Health 40:1-30

Moonmoon M, Uddin MN, Ahmed S, Shelly NJ, Khan MA (2010) Cultivation of different strains of king oyster mushroom (Pleurotus eryngii) on saw dust and rice straw in Bangladesh. Saudi J Biol Sci 17:341-345

Sánchez C (2010) Cultivation of Pleurotus ostreatus and other edible mushrooms. Appl Microbiol Biotechnol 85:1321-1337

Streit E, Schatzmayr G, Tassis P, Tzika E, Marin D, Taranu I, Tabuc C, Nicolau A, Aprodu I, Puel O, Oswald IP (2012) Current situation of mycotoxin contamination and co-occurrence in animal feed-focus on Europe. Toxins (Basel) 4:788-809

Upadhaya SD, Park MA, Ha JK (2010) Mycotoxins and their biotransformation in the rumen: a review. Asian Aust J Anim Sci $23: 1250-1260$

Zain ME (2011) Impact of mycotoxins on humans and animals. J Saudi Chem Soc 15:129-144

Zhang R, Li X, Fadel JG (2002) Oyster mushroom cultivation with rice and wheat straw. Bioresour Technol 82:277-284 